\title{
Políticas Públicas Educativas y aseguramiento de la calidad en la Educación Superior ${ }^{1}$
}

\author{
Public Education Policies and Quality Assurance in Higher Education \\ Políticas Públicas Educacionais e garantia da qualidade da Educação \\ Superior
}

Luis Alberto Malagón Plata ${ }^{2}$

Universidad del Tolima, Colombia

Luz Helena Rodríguez Rodríguez ${ }^{3}$

Universidad del Tolima, Colombia

Diego Fernando Machado Vega ${ }^{4}$

Universidad Surcolombiana, Colombia

Recepción: 03/12/2017

Evaluación: 30/01/2018

Aceptación: 23/02/2018

Artículo de Investigación - Revisión

https://doi.org/10.19053/01227238.4999

\section{RESUMEN}

El presente artículo da cuenta de los resultados de la investigación que analizar los mecanismos que componen los sistemas de aseguramiento de la calidad de la educación superior en el contexto de América Latina, haciendo énfasis en Colombia, para resaltar las tendencias cu- rriculares y académicas que se consolidan institucionalmente ante las exigencias y problemáticas que presentan los nuevos mercados laborales en la globalización capitalista, como reflejo de la implementación de las actuales políticas públicas educativas. A través del estudio de caso

1 El presente artículo es producto del proyecto de investigación titulado "Tendencias del currículo en las políticas de aseguramiento de la calidad de la educación superior en América Latina. Estudio de caso: Chile, Costa Rica, México, Colombia”, presentado y desarrollado por el grupo de investigación en currículo, Universidad y Sociedad. Financiado por la Oficina de Investigaciones y Desarrollo Científico de la Universidad del Tolima. Código 410111

2 Doctor en Educación de la universidad de Costa Rica. Docente de Planta tiempo completo, adscrito al Departamento de Psicopedagogía de la Facultad de ciencias de la Educación de la Universidad del Tolima. Investigador del grupo de Investigación en Currículo, Universidad y Sociedad. Correo electronico: lmalagon@ut.edu.co

3 Magister en Educación de la Universidad del Tolima. Docente de planta tiempo completo, adscrita al Departamento de Estudios Interdisciplinarios del Instituto de Educación a Distancia de la Universidad del Tolima. Investigador del grupo de Investigación en Currículo, Universidad y Sociedad. Correo electronico: 1rodriguez@ut.edu.co Enviar correspondencia a este autor.

4 Magister en Educación de la Universidad del Tolima. Docente ESAP-Territorial Huila, Caquetá, Putumayo.Correo electronico: dfmachadov@gmail.com 
en Colombia y la revisión de documentos institucionales, se encuentra que la educación superior y las políticas públicas de aseguramiento de la calidad aparecen como el dispositivo de funcionamiento de una estructura asimétrica internacional. En el caso colombiano, más que dificultades en la formulación, se encuentran problemas en las fases de implementación y ejecución, debido a la inflexibilidad de las culturas académicas y administrativas de las instituciones de educación superior. Finalmente se concluye que el aseguramiento de la calidad y la acreditación contribuyen con la generación y organización de información para los sistemas de educación superior. Las políticas públicas de calidad y de internacionalización, en el sistema de educación superior colombiano, dependen del logro de niveles aceptables y productivos de consolidación, y de maniobrabilidad de la autoridad directiva y docente; en medio de una cultura académica de calidad, administrativa, e institucional de gobernabilidad favorable.

Palabras clave: Políticas publicas educativas; Calidad de la educación; aseguramiento de la calidad; Educación superior; Capitalismo académico.

\section{ABSTRACT}

This article reports on the results of the research that analyzes the mechanisms that shape the quality assurance systems of higher education in the context of Latin America. With special emphasis on Colombia, we try to highlight the, institutionally consolidated, curricular and academic trends emerged from the problems and demands of the new labor markets, framed in the capitalist globalization, as a reflection of the currently implemented public education policies. Through the case study in Colombia and the review of institutional documents, it was found that higher education and public quality assurance policies appear as the operating device of an asymmetric international structure. In the case of Colombia, rather than difficulties in the formulation, problems are found in the implementation and execution phases, due to the inflexibility of the academic and administrative cultures of higher education institutions. Finally, it is concluded that quality assurance and accreditation contribute to the generation and organization of information for higher education systems. Public policies of quality and internationalization, in the Colombian higher education system, depend on the achievement of acceptable and productive levels of consolidation and the management of the directive and teaching authority in the midst of a quality, administrative, and institutional academic culture of favorable governance.

Keywords: Public education policies; Quality of education; Quality assurance; Higher education; Academic capitalism.

\section{RESUMO}

Apresenta-se os resultados da pesquisa que busca analisar os mecanismos que compõe os sistemas de controle da qualidade da educação superior no contexto da América Latina, com ênfase na Colômbia, para ressaltar as tendências curriculares e acadêmicas que se consolidam institucionalmente frente as exigências e problemáticas que apresentam os novos mercados de trabalho na globalização capitalista, como reflexo da implementação das atuais políticas públicas educacionais. Por meio deste estudo de caso na Colômbia e da revisão de documentos institucionais, se conclui que a educação superior e as políticas públicas de controle da qualidade aparecem como o dispositivo de funcionamento de uma estrutura assimétrica internacional. No caso colombiano, mais que dificuldades na formulação, encontram-se problemas nas fases de implementação e execução, devido à inflexibilidade das culturas acadêmicas e administrativas das instituições de educação superior. Finalmente, se conclui que o controle de qualidade e o reconhecimento contribuem com a geração e organização 
da informação para os sistemas de educação superior. As políticas públicas de qualidade e de internacionalização no sistema de educação superior colombiano dependem de se alcançar níveis aceitáveis e produtivos de consolidação e de manobrabilidade da autoridade diretiva e do- cente; em meio a uma cultura acadêmica de qualidade, administrativa e institucional de governabilidade favorável.

Palavras-chave: Políticas públicas educacionais; Qualidade da educação; controle da qualidade; Educação superior; Capitalismo acadêmico.

\section{INTRODUCCIÓN}

El escenario latinoamericano actual de cambios curriculares a causa de las demandas sociales y productivas de la sociedad del conocimiento se pretende encarar con la implementación de los sistemas de aseguramiento de la calidad que se relacionan con los procesos de ajuste institucional, tanto de la oferta académica, en cuanto al número y condiciones de los programas, universidades y proveedores de servicios educativos, como de la administración y gestión de las instituciones de educación superior, además de la capacidad de flexibilidad institucional para enfrentar y adecuarse a las demandas de la sociedad y del entorno en general.

De hecho, los ajustes institucionales en cuanto a la oferta académica, a la administración y gestión de las universidades y a la flexibilidad institucional para atender las demandas académicas y de conocimiento de la sociedad, son el resultado del avance de una reflexión oficial sobre la pertinencia social y laboral de la educación superior, que, no obstante, enfrenta un conjunto de críticas y debates frente al carácter de formalización y de homogenización de los procesos de aseguramiento de la calidad son, "sistemas centrados en procesos e insumos, con fuerte énfasis en la homogenización y estandarización, mediante el cumplimiento de determinadas condiciones y factores comunes a todos los programas e instituciones" ${ }^{\prime \prime}$.

Es éste, el sentido que permite analizar el contexto latinoamericano como una dimensión relacional y objetiva, de la que es posible apreciar el establecimiento de un conjunto de procesos institucionales comunes, que están sustentados política e históricamente, en el marco de las macro-políticas de apertura económica, política y social iniciados en la década de los noventa del siglo pasado, que tienen como objetivo el ajuste de los mercados públicos y privados nacionales, en conjunción con la internacionalización de la provisión y racionalización del mercado de servicios, a través de un escenario que se caracteriza por la puesta en marcha de variados sistemas de aseguramiento de la calidad de la educación superior en los distintos países latinoamericanos, donde en el caso de Colombia, posee un modelo fundamentado en la evaluación y acreditación de programas, así como en la acreditación de las instituciones de educación supe-

5 Víctor Gómez, Jorge Celis, "El Sistema de Aseguramiento de la Calidad de la Educación Superior: Consideraciones Sobre la Acreditación en Colombia", Revista Colombiana de Sociología, vol. 32, no. 2 (2009): 86. doi.org/10.15446/rcs 
rior, complementado con la imposición de estándares mínimos de calidad para el estricto funcionamiento y oferta de los programas o carreras profesionales.

Por lo anterior, en la presente investigación se analiza el conjunto de mecanismos que componen los denominados sistemas de aseguramiento de la calidad de la educación superior en el contexto de América Latina, haciendo énfasis en Colombia, con el propósito de resaltar las tendencias curriculares que se consolidan institucionalmente como respuestas académica a las exigencias y a las problemáticas que presentan los nuevos mercados laborales en el ámbito de la globalización capitalista, como reflejo de la implementación de las actuales políticas públicas educativas que son expresión del fortalecimiento de los intereses del denominado Estado evaluador contemporáneo. En el ámbito de lo metodológico, se acude al análisis documental y de contenido de diversos documentos provenientes de los entes estatales relacionados con la educación superior, con la intención de elaborar y complejizar un hecho de estudio, que, a la vez, que es conflictivo y problemático por la diversidad de racionalidades e intereses en interacción, se prolonga y extiende por distintos marcos institucionales, legales y de participación, en el que se superponen diversidad de entes y jurisdicciones públicas para su ejecución.

Respecto al estado del arte, se resaltan los estudios e investigaciones en el campo de las políticas públicas educativas de aseguramiento de la calidad e internacionalización. En relación a las políticas públicas educativas, se resaltan algunos ámbitos generales de análisis que se centran en la producción de información sobre algunos factores primordiales de los sistemas de educación superior. De esta manera, se destacan las investigaciones sobre las repercusiones de los proyectos de políticas educativas, ya sea, en sus fases de formulación, implementación o de evaluación, que son los tópicos más estudiados relativos a políticas sociales ${ }^{6}$; de igual forma, se resaltan las estudios enmarcadas en la teoría crítica y la teoría funcionalista, que buscan dimensionar de las políticas educacionales desde tres perspectiva diferentes; la retórica de la política, la política impulsada legalmente y la política que se implementa en la práctica ${ }^{7}$, las cuales son distinciones aparentes de un mismo hecho, que pueden entenderse en relación a la educación, de la siguiente forma; "la retórica política se refiere a enunciados amplios de metas educacionales que a menudo pueden ser encontrados en discursos nacionales de líderes políticos. Las políticas consagradas legalmente son los decretos o leyes que definen estándares explícitos y orientaciones para el sector educativo. Las políticas efectivamente implementadas son aquellas legalmente impulsadas, modificadas o sin modificar, que se traducen en acciones concretas a través de cambios sistemáticos y programados" ${ }^{8}$.

6 Jaime Moreles. "Uso de la investigación en la toma de decisiones políticas. Una aproximación a su estudio", Revista Archivos Analíticos de Políticas Educativas, vol. 17, no. 13 (2009): 1-25. En http://epaa.asu.edu/ojs/article/viewFile/15/15

7 Oscar Espinosa. "Reflexiones sobre los conceptos de política, políticas públicas y política educacional". Revista Archivos Analíticos de Políticas Educativas, vol. 17, no. 8 (2009): 1-13. En http://epaa.asu.edu/ojs/article/viewFile/10/10

8 Alberto Espinosa, Empresarios y política pública educativa (Revista Dinero.com. 2009) http://www.dinero.com/edicion-impresa/ columnistas/empresarios-politica-publica-educativa_61202.aspx (23 octubre de 2014) 
Se resaltan investigaciones que se enfocan en la producción de información en general, en relación al sistema de educación superior, sobre la utilización conceptual que diferentes tipos de usuarios hacen de las investigaciones, en vista de alcanzar panorámicas sobre los asuntos públicos de la educación, así como para generar consensos sobre directrices políticas en el sector, y para propiciar una adecuada recepción de parte de los funcionarios de las distintas opciones y alternativas para la toma de decisiones ${ }^{9}$, en el marco de las instituciones relacionadas con la educación universitaria.

Por su parte, otro ámbito de investigaciones en el marco de las políticas públicas educativas, que también se centra en la producción de información para el sistema de educación superior, pero, ahora, en el sentido del análisis de la población de intervención pública, son los estudios sobre la focalización de las políticas educativas de nivel superior, en relación a los créditos financieros para las familias de medios y bajos recursos, que han sacado a la luz pública, la depreciación de la calidad del servicio educativo público, la precariedad subyacente en la infraestructura, en recursos metodológicos, tecnológicos y presupuestarios, así como en el deterioro de las condiciones de trabajo de los docentes ${ }^{10}$.

Otros estudios se enfocan en la evolución positiva de la cobertura de la educación superior, resaltándose de los países latinoamericanos, la profundización de la notable desigualdad entre la población, frente al acceso a los niveles de formación superior ${ }^{11}$. Entre tanto, se indaga sobre los factores que obstruyen la interacción entre la investigación y sus usuarios, de cómo acceden, seleccionan y utilizan los resultados de investigación, en referencia a la heterogeneidad de la comunidad académica y científica, que ha ocasionado las subdivisiones en innumerables grupos, y que fragmenta los intereses de investigación ${ }^{12}$; situación que en el ámbito académico, se conoce como los análisis sobre las capacidades de investigación de sistemas o instituciones particulares de educación superior.

Por otra parte, en relación a las investigaciones sobre la gerencia y la producción de información especializada para los sistemas de educación superior, para comenzar, se resaltan algunas temáticas, aún muy cercanas a las del ámbito anterior, en relación a los análisis y las descripciones de las dificultades de acceso a la investigación y a la instauración de estrategias y alternativas para facilitar y promover la utilización e interacción de la información por parte de los grupos de usuarios, especialmente, los investigadores y los tomadores de decisiones del sector educativo.

Otras investigaciones se enfocan en los mecanismos de acreditación institucional, analizados como dispositivos con el propósito de contribuir con más y

$9 \quad$ Jaime Moreles. “Uso de la investigación en la toma de decisiones políticas. Una aproximación a su estudio", Revista Archivos Analíticos de Politicas Educativas, vol. 17, no. 13 (2009): 1-25. En http://epaa.asu.edu/ojs/article/viewFile/15/15

10 Pablo Aparicio. "Educación y jóvenes en contextos de desigualdad socioeconómica", Revista Tendencias y perspectivas en América Latina. Archivos Analíticos de Políticas Educativas, vol. 17, no. 12 (2009): 1-35. En http://epaa.asu.edu/ojs/article/viewFile/14/14

11 José Martín. "Los vínculos entre capital humano y la competitividad en América Latina". Revista Estudios Económicos de Desarrollo Internacional, no. 002 (2007): 82-102. En http://redalyc.uaemex.mx/pdf/165/16570204.pdf

12 Jaime Moreles. “Uso de la investigación en la toma de decisiones políticas. Una aproximación a su estudio", Revista Archivos Analíticos de Políticas Educativas, vol. 17, no. 13 (2009): 1-25. En http://epaa.asu.edu/ojs/article/viewFile/15/15 
mejor información a los sistemas de educación superior, ya que, dichos sistemas, regulados por sí solos, son susceptibles de presentar múltiples asimetrías de información ${ }^{13}$ lo cual, se constituye en un hecho importante, en el escenario de las macro-políticas educativas, que comparten y compiten por las asignaciones de recursos en el ámbito de las organizaciones multinacionales e internacionales.

Es trascendental resaltar, que la investigación en el ámbito de la gerencia y la producción de información especializada, es un tema de gran importancia en la actualidad, que manifiesta su consolidación en la emergencia de instituciones y entes institucionales consagrados únicamente a esta actividad, como los son el Laboratorio de Análisis Institucional del Sistema Universitario Mexicano (LAISUM), que tiene como propósito la gerencia de información de muy variada procedencia; "notas periodísticas, documentos de organismos nacionales e internacionales, bancos especializados de fichas bibliográficas, y una relación precisa de ligas de interés, además de una variedad de documentos de cada una de las universidades públicas mexicanas ${ }^{\prime 14} \mathrm{O}$, como los son las Oficinas de Análisis Institucional (OAI) de diversas universidades del sistema de educación superior chileno, que consolidan su posición a través de convertirse en un importante punto de contacto entre las universidades y su entorno, en una relación que se da en dos sentidos; en el que las Oficinas de Análisis Institucional proveen información oficial a las instancias externas que lo requieran, y en el que la OAI proveen a la organización de información del entorno en que se desenvuelven ${ }^{15}$

En otro aspecto, se tienen en cuenta las investigaciones particulares en torno a las políticas públicas educativas, en el ámbito del aseguramiento de la calidad, de las que se resaltan los análisis sobre el impacto de las políticas de calidad en contextos y escenarios en el que participan distintos actores con inclinaciones diferentes; existiendo intereses variados que pueden ir desde el genuino interés por el mejoramiento o el direccionamiento del sistema hacia una mayor transparencia, hasta el solo propósito de obtener una certificación pública en contextos de mercado o bien limitar lo que puede ser una excesiva diversidad ${ }^{16}$.

Además, sobre estas mismas problemáticas, es importante tener en cuenta, que en el escenario internacional, en relación a las metodologías e instrumentos de investigación, se presentan una gran variedad de enfoques y aproximaciones que hace difícil definir operativamente el impacto de los procesos de aseguramiento de la calidad sobre los sistemas y las instituciones de educación superi-

13 Gonzalo Zapata, Ivo Tejada. "Impactos del aseguramiento de la calidad y acreditación de la educación superior. Consideraciones y proposiciones", Revista Calidad de la Educación, no. 31 (2009): 192-209. En https://www.calidadenlaeducacion.cl/index.php/rce/article/download/168/174

14 Carmen Zambrano, Gonzalo Benítez. "Laboratorio de análisis institucional del sistema universitario mexicano (LAISUM)". Revista Perfiles Educativos, vol. 32, no. 129 (2010): 184-186. En http://www.iisue.unam.mx/seccion/perfiles/

15 Felipe Rivera, Pablo Astudillo, Enrique Fernández. "Información y toma de decisiones: Oficinas de análisis institucional (OAI)". Revista Calidad de la Educación, no. 30 (2009): 162-178. En http://www.cned.cl/public/Secciones/seccionpublicaciones/doc/63/ cse_articulo799.pdf

16 Gonzalo Zapata, Ivo Tejada. "Impactos del aseguramiento de la calidad y acreditación de la educación superior. Consideraciones y proposiciones", Revista Calidad de la Educación, no. 31 (2009): 192-209. En http://www.cned.cl/public/Secciones/seccionpublicaciones/doc/64/cse_articulo839.pdf 
or ${ }^{17}$, hecho que complica el ámbito de los análisis comparativos, pero, que sin duda, enriquece los marcos de análisis diferenciados entre los distintos países.

Finalmente, antes de terminar, es de resaltar de las investigaciones y estudios sobre políticas de internacionalización de los sistemas educativos, los análisis que se enfocan a medir las repercusiones de los proyectos promovidos por organizaciones internacionales en distintos países, para encauzar la generación de investigaciones en diferentes ámbitos educativos; sin embargo, en este campo, las investigaciones que se privilegian por los organismos internacionales, son las que se reducen a los análisis correlaciónales entre variables de competitividad y de capital humano, en las cuales, se observa la evolución de la dotación humana, medida por el nivel educativo de la población, y de la posición competitiva, medida por las cuotas de mercado de los países ${ }^{18}$, en este caso, los países latinoamericanos.

Se presenta a continuación el desarrollo teórico y la construcción de un referente conceptual donde se circunscriben los contornos de las tendencias, reflexiones, debates y críticas, relacionados a los sistemas de aseguramiento de la calidad en la educación superior en el marco latinoamericano enfatizando en Colombia, destacando las tendencias curriculares que se consolidan institucionalmente como respuestas a las exigencias de los nuevos mercados laborales en el ámbito de la globalización capitalista, como reflejo de la implementación de las actuales políticas públicas educativas.

\section{Políticas públicas educativas}

Las políticas públicas se relacionan con un conjunto de decisiones interrelacionadas que son adoptadas por un actor o grupo de actores políticos que involucran la definición de metas y medios para su logro en el marco de una situación particular; son un conjunto de opciones colectivas interdependientes que se asocian a decisiones que adoptan los gobiernos y sus representantes. ${ }^{19}$

En el contexto colombiano, las políticas públicas en general y las políticas públicas educativas en particular, son temas de debate actual, gracias en gran medida a la difusión, internalización e interpretación que los distintos sectores, grupos y movimientos sociales y políticos, han logrado de las políticas de participación y acción ciudadana, que tienen su germen en la Constitución de 1991, $y$, que se ven complementadas académicamente por la docencia e investigación

17 Judith Scharager, María Aravena. "Impacto de las políticas de aseguramiento de la calidad en programas de educación superior: Un estudio exploratorio". Revista Calidad de la Educación, no. 32 (2010): 16 - 42. En http://www.cned.cl/public/Secciones/seccionpublicaciones/doc/67/cse_articulo899.pdf

18 José Martín. "Los vínculos entre capital humano y la competitividad en América Latina”. Revista Estudios Económicos de Desarrollo Internacional, no. 002 (2007): 82-102. En http://redalyc.uaemex.mx/pdf/165/16570204.pdf

19 Oscar Espinosa. "Reflexiones sobre los conceptos de política, políticas públicas y política educacional", Archivos Analíticos de Políticas Educativas, vol. 17 no. 8 (2009): 3. En http://epaa.asu.edu/ojs/article/viewFile/10/10. Diana Elvira Soto Arango. "Legislación e imaginarios sociales en el escalafón y los salarios de los educadores de primaria en Colombia 1952 - 1994" Revista Historia de la Educación Latinoamericana no. 21 (2013): 229-262. 
en el área del análisis de las políticas públicas y de la gestión pública que llevan a cabo algunas de las instituciones de educación superior del país.

\section{Políticas y de programas de autorregulación de la demanda y de la oferta educativa}

Por otra parte, en relación a las políticas públicas educativas, se aprecia un conjunto complementario de políticas y de programas de autorregulación de la demanda y de la oferta educativa, que se articulan en una serie de planes y proyectos de mercadeo e internacionalización de la educación superior. Tales políticas y programas, se componen, de una parte, de una variedad heterogénea de micro-políticas de desmonte del subsidio a la oferta; es decir, del financiamiento de las universidades con recursos del presupuesto público; por un sistema de créditos y financiamiento vía demanda, bajo el desarrollo de políticas de focalización del gasto ${ }^{20}$, que se sustentan públicamente, en el discurso a las familias de estratos medios, en relación al gasto en educación superior como la forma de inversión en capital humano a largo y mediano plazo.

Por otro lado, de una macro-política de nivel institucional en todas sus jerarquías, que se dirige a la organización y gerenciamiento de la información y el conocimiento, como un insumo y elemento fundamental del trabajo académico, pues, cumplen un rol multifuncional: son recursos para la toma de decisiones y la resolución de problemas asociados a la investigación, la docencia y la gestión académica ${ }^{21}$, que son los elementos y los factores que regulan la oferta educativa del sistema de educación superior en general, y que proporcionan posición, recursos e incentivos a las instituciones de educación superior en particular.

Así, estos programas de autorregulación de la demanda y de la oferta educativa, se articulan en planes y proyectos de mercadeo e internacionalización de la educación superior, a través de iniciativas interinstitucionales como las de Empresarios por la Educación, Fe y Alegría y la Fundación Ford, que buscan por medio de alianzas nacionales e internacionales ofrecer alternativas de cambio estructural a los sistemas educativos ${ }^{22}, \mathrm{o}$, a través de definiciones operativas de internacionalización que son desarrolladas por grupos de cooperación institucional y universitario como la RED ALMA MATER.

\section{Componentes de las políticas públicas educativas}

Son los referidos al conjunto de decisiones interrelacionadas y al conjunto de opciones colectivas, que son precisamente, los elementos claves sobre los que se

20 Oliver Mora. "Las políticas educativas en América Latina: Un análisis de la educación superior desde la visión de la banca multilateral". Apuntes del CENES, vol. 25, no. 40 (2005): 249-262. En http://aplica.uptc.edu.co/Publicaciones/ApuntesCenes/Documentos/170_24273_12.pdf

21 Uriel Giraldo, Darío Abad, Edgar Díaz, Bases para una política de calidad de la educación superior en Colombia (Bogotá: CNA, 2002) http://www.cna.gov.co/1741/articles-186502_doc_academico10.pdf (6 de septiembre de 2013)

22 Oscar Espinosa. "Reflexiones sobre los conceptos de política, políticas públicas y política educacional”, Archivos Analíticos de Políticas Educativas, vol. 17 no. 8 (2009): 3. En http://epaa.asu.edu/ojs/article/viewFile/10/10 
ponen en juego los intereses de los sistemas educativos nacionales en el escenario de la internacionalización del mercado educativo, que, como ejes del sistema, son a los que apuntan los organismos internacionales como el Banco Mundial, cuando proponen recomendaciones como la de la integración eficiente de los sistemas educativos a nivel global, a través de la introducción y el uso público y cotidiano de un lenguaje economicista en el sector, en el sentido de conceptos tales como capitalismo académico, universidad emprendedora, base de financiamiento discrecional, entre otros ${ }^{23}$.

\section{El capitalismo académico en las políticas públicas educativas}

De este modo, el capitalismo académico, puede entenderse, como la explotación intensiva del capital humano, formado por académicos vinculados a las universidades, con potencial de fomentar conocimiento, capaz de atraer los intereses de representantes del sector productivo ${ }^{24}$; el cual, a su vez, a través de los distintos mecanismos por los que se puede acumular capital humano (educación formal, formación continua, formación ocupacional) entra a jugar un papel decisivo en la estrategia de fomento de la competitividad, tanto en el ámbito macroeconómico de los países, como en el ámbito microeconómico de las empresas ${ }^{25}$.

De ahí que, de las políticas públicas educativas que se articulan al escenario del capitalismo académico, se resalte la consolidación general en los países latinoamericanos, de las políticas de aseguramiento de la calidad de la educación, y, dentro de éstas, de manera particular, de las políticas de acreditación de la educación superior. Las políticas de aseguramiento de la calidad son el conjunto de procedimientos, normas y prácticas, internas y externas a las instituciones, destinadas a la mantención y promoción de estándares de calidad; y, la acreditación, es una forma particular de política de aseguramiento de la calidad, que privilegia procedimientos de evaluación permanente asociados a la verificación y certificación pública de criterios de calidad ${ }^{26}$.

A su vez, como política complementaria en el entorno del capitalismo académico a las de aseguramiento de la calidad, se encuentran las políticas de análisis institucional, que consolidan su función, a través del trabajo continuo de gerencia y sistematización de la información pertinente para los sistemas de educación superior, las cuales, se concretan como organismos de apoyo, suste-

23 Ernesto Villanueva. "Perspectivas de la educación superior en América Latina: Construyendo futuros", Revista Perfiles Educativos. no. 129 (2010): 86-101. En http://www.iisue.unam.mx/seccion/perfiles/

24 Manolita Correia. "La internalización de la industria de la educación: El caso Australiano", Revista Calidad de la Educación, no. 32 (2010): 170-204. En http://www.cned.cl/public/secciones/seccionpublicaciones/doc/67/cse_articulo904.pdf

25 José Arnaiz. “La Interrelación entre el Nivel Educativo y la Posición Competitiva en América Latina y la Unión Europea”, Anales de Estudios Económicos y Empresariales, no. 20 (2010): 87. En https://dialnet.unirioja.es/descarga/articulo/3763134.pdf

26 Zapata, Gonzalo y Tejada, Ivo. "Impactos del aseguramiento de la calidad y acreditación de la educación superior. Consideraciones y proposiciones”, Revista Calidad de la Educación, no. 31 (2009): 192-209. En http://www.cned.cl/public/Secciones/seccionpublicaciones/doc/64/cse_articulo839.pdf 
nto y orientación, tanto para los sistemas universitarios nacionales, o como las distintas oficinas de análisis institucional de las universidades.

Así, la característica predominante que adquieren los sistemas de educación superior a través de las políticas públicas educativas sobresalientes en la actualidad, es, según Fernández ${ }^{27}$, el del proceso de descapitalización de la universidad pública que conduce a dos condiciones; el de un modelo de gobierno y de gestión orientados hacia la coordinación de los sistemas nacionales vía mercado, y el del desarrollo de políticas de financiamiento orientadas hacia la administración descentralizada (financiamiento de los clientes) y a los resultados de desempeño.

\section{Políticas Públicas de Internacionalización y Políticas Públicas de Calidad}

Como se anotó anteriormente, desde la década de 1990 la tendencia a la expansión e integración internacional de la educación superior se ha generaliza$\mathrm{do}^{28}$, a través, de la consolidación, acoplamiento y difusión mundial de las políticas públicas de internacionalización y de aseguramiento de la calidad, que en el transcurso de dos décadas, se han convertido en un dispositivo de configuración del sistema-mundo del capitalismo académico, que en pocas palabras:

Es la materialización de la situación de dependencia en el espacio universitario, donde la economía del conocimiento de cierto grupo de países está condicionada por el desarrollo y expansión de la economía del conocimiento de otros países, ante los cuales, los primeros se someten y se subordinan, generando una situación de atraso. $^{29}$

Situación compleja, que manifiesta una estructura del sistema educativo mundial con fuertes rasgos de asimetrías en el mercado internacional de la educación superior entre países del primer y del tercer mundo, que se expresa de manera contundente y contradictoria, en las agresivas políticas de atracción de estudiantes extranjeros y de exportación de servicios educativos por parte de los países desarrollados, que, como en el caso de Australia, presenta un progresivo desequilibrio en el crecimiento de la matrícula entre nacionales y extranjeros, pues, de 1995 a 2001 el número de estudiantes australianos que ingresan en la educación terciaria aumentó un $8.6 \%$, en contrapartida al número de extranjeros que ingresan a su sistema educativo superior, que aumentó en 146\%; lo cual, es una situación de imperfección de mercado, que conduce a una dependencia del sistema en relación a la matrícula internacional, que, además, da indicios de una propensión al acrecentamiento, en el momento en que los gobernantes

27 Eduardo Fernández. "El sistema-mundo del capitalismo académico: Procesos de consolidación de la universidad emprendedora". Revista Archivos Analiticos de Políticas Educativas. no. 21 (2009): 1-43. En http://epaa.asu.edu/ojs/article/viewFile/387/511

28 Felipe Rivera, Pablo Astudillo, Enrique Fernández. "Información y toma de decisiones: Oficinas de análisis institucional (OAI)", Revista Calidad de la Educación, no. 30 (2009): 164. En: http://www.cned.cl/public/Secciones/seccionpublicaciones/doc/63/cse_articulo799.pdf

29 Eduardo Fernández. "El sistema-mundo del capitalismo académico: Procesos de consolidación de la universidad emprendedora", Revista Archivos Analíticos de Políticas Educativas, no. 21 (2009): 4. En http://epaa.asu.edu/ojs/article/viewFile/387/511 
australianos reconocen una creciente reducción en el número de estudiantes que terminan la enseñanza media en este país. ${ }^{30}$

Así, frente a este continuo desequilibrio del mercado mundial de la educación superior, las políticas públicas de aseguramiento de la calidad aparecen como el dispositivo de funcionamiento de esta estructura asimétrica internacional, al disponer a las instituciones de educación superior de los países subdesarrollados, al cumplimiento de los estándares de calidad creados y funcionales del primer mundo, a través de lo que se conoce como el modelo de acreditación institucional, que se plantea como una auditoría académica, con un tipo de criterios que presentan un claro acento hacia la definición e implementación de una serie de políticas y mecanismos de aseguramiento de la calidad en el desarrollo de la gestión institucional y de la docencia, en busca de impactar en el mejoramiento continuo de las instituciones a través del afianzamiento del aseguramiento interno, de la exigencia de líneas básicas de calidad y de capacidades instaladas de autorregulación ${ }^{31}$.

\section{En el contexto del capitalismo académico de nivel global}

El continuo fortalecimiento de la competencia internacional por la trascendencia del desarrollo de la investigación en ciencia y tecnología y de la permanente cualificación de los expertos adscritos a las instituciones de educación superior, trae como consecuencia, la incapacidad del Estado para financiar los altos costos de las exigencias en calidad de la oferta educativa y del fomento y consolidación de la investigación científica, por lo cual, frente a la necesidad de ser competitivos y de que las universidades conserven el prestigio conquistado, el Estado adopta políticas con menos reglamentos en el sector educativo para favorecer su explotación comercial ${ }^{32}$.

De ahí que, las motivaciones económicas, se convierten en incentivos a la cultura docente y administrativa de las instituciones de educación superior, para impulsar los cambios necesarios en el sistema organizativo, de modo que se movilice y estimule el trabajo por el mejoramiento de los programas, estableciendo gradualmente una cultura de la evaluación en un medio de creciente competencia, incorporando en la práctica educativa espacios de trabajo para desarrollar propuestas de mejoras de planes de estudio y de innovación de recursos de enseñanza, para así, optimizar los indicadores de eficiencia ${ }^{33}$.

30 Manolita Correia. "La internalización de la industria de la educación: El caso Australiano", Revista Calidad de la Educación, no. 32 (2010): 170-204. En http://www.cned.cl/public/secciones/seccionpublicaciones/doc/67/cse_articulo904.pdf

31 Gonzalo Zapata, Ivo Tejada. "Impactos del aseguramiento de la calidad y acreditación de la educación superior. Consideraciones y proposiciones", Revista Calidad de la Educación, no. 31 (2009): 200. En http://www.cned.cl/public/Secciones/seccionpublicaciones/ doc/64/cse_articulo839.pdf

32 Manolita Correia. "La internalización de la industria de la educación: El caso Australiano", Revista Calidad de la Educación, no. 32 (2010): 170-204. En http://www.cned.cl/public/secciones/seccionpublicaciones/doc/67/cse_articulo904.pdf

33 Judith Scharager, María Aravena. "Impacto de las políticas de aseguramiento de la calidad en programas de educación superior: Un estudio exploratorio". Revista Calidad de la Educación, no. 32 (2010): 16 - 42. En http://www.cned.cl/public/Secciones/seccionpublicaciones/doc/67/cse_articulo899.pdf 
Es decir, las instituciones universitarias, al enfrentar las reformas al sistema de educación en relación a las políticas de mercado y de regulación de la calidad, se ven atacadas y desplazadas de su legitimidad social, en cuanto a la pérdida del monopolio de la producción del conocimiento avanzado, en relación al detrimento del control del valor simbólico y de cambio de las credenciales que otorga en grados académicos y diplomas profesionales, $y$, finalmente, en cuanto a la pérdida de su capacidad de autorregulación interna, que pasa a agencias oficiales y de mercado, el control y regulación de sus procesos y resultados, que trae como consecuencia, el cambio de los derechos atribuidos a las universidades como portadoras del poder intelectual ${ }^{34}$.

\section{Transformaciones en la relación universidad, mercado y estado}

Con la adaptación de mecanismos de gestión y de dirección de la empresa privada en las instituciones de educación superior públicas, la funcionalidad institucional hacia el conocimiento y su articulación a los intereses de la sociedad se desplazan en su misión, entendiéndose desde ahora la educación universitaria, como el sector cuya misión principal sería la transmisión de datos e información en pro del crecimiento de la ganancia, de manera que constituya una industria y no un derecho ${ }^{35}$; contexto en el cual, la reacción de las instituciones de educación superior frente a los indicios y las exigencias del mercado de la educación en la actualidad, se enfocan en dos tendencias de reestructuración institucional.

Por una parte, frente al mercado interior de la educación superior y sustentadas en las ley 749 de 2002, que proporciona legitimidad social y laboral a la formación por ciclos técnico, tecnológico y profesional ${ }^{36}$, las instituciones redefinen sus estructuras académicas y administrativas, para adoptar la modalidad por ciclos propedéuticos, de manera que se responda con competencias diferenciadas a los distintos niveles de complejidad de las demandas laborales del país.

A su turno, ante los desafíos y las complejidades que demanda el mercado global de la educación superior, las instituciones universitarias sustentadas en su autonomía académica, administrativa y gestionaría, parten de la adopción de los principios de la internacionalización del currículo, que buscan la orientación de las acciones de la dimensión académica, al afianzamiento de las competencias globales en los estudiantes, de la movilidad de los estudiantes y de los docentes, de las estrategias de bilingüismo, y de bibliotecas como plataformas a la socie-

34 Eduardo Fernández. "El sistema-mundo del capitalismo académico: Procesos de consolidación de la universidad emprendedora". Revista Archivos Analíticos de Políticas Educativas, no. 21 (2009): 1-43. En http://epaa.asu.edu/ojs/article/viewFile/387/511

35 Oliver Mora. "Las políticas educativas en América Latina: Un análisis de la educación superior desde la visión de la banca multilateral”. Apuntes del CENES, vol. 25, no. 40 (2005): 249-262. En http://aplica.uptc.edu.co/Publicaciones/ApuntesCenes/Documentos/170_24273_12.pdf

36 Elvia González, Sandra Duque. "Sobre el marco normativo para formular una propuesta de políticas públicas para la formación por ciclos y la evaluación por competencias en la educación superior colombiana”, Revista Opinión Jurídica, no. 14 (2008): 83-98. En http://redalyc.uaemex.mx/redalyc/pdf/945/94512646006.pdf 
dad de la información y el conocimiento ${ }^{37}$, para responder de forma adecuada y competitiva a los retos de un mundo laboral, que ante los permanentes cambios de competencias profesionales derivados de las transformaciones de la ciencia y la tecnología, exigen una readecuación estructural y académica de las instituciones universitarias, para que se comprometan con la formación integral.

\section{Contexto latinoamericano de exigencias curriculares en la educación superior}

Los procesos de aseguramiento de la calidad influyen en el funcionamiento de las instituciones de educación superior y en el desarrollo curricular, a través de la creciente intervención oficial en la formalización y regulación de la calidad, que tiene como propósito incentivar de manera diferenciada, por una lado, a las universidades, para que tomen la iniciativa en el establecimiento de procedimientos que conduzcan a la consecución de altos estándares de calidad y a la internacionalización de la educación superior, y, por otro lado, a los proveedores de servicios educativos, a que ajusten su oferta académica según los estándares mínimos de calidad impuestos por los organismos competentes.

De ahí que, los procesos de mejoramiento de la calidad, exijan en el nivel interno de las instituciones de educación superior, sobre todo en las universidades, de nuevos modelos de gestión y de organización institucional, que revitalicen los aquejados y desgastados procedimientos de la burocracia y de la jerarquía tradicional, para que un creciente nivel de gobernabilidad en las universidades se convierta en un factor de ejecución y de coordinación institucional para el impulso a los planes de mejoramiento continuo de la calidad, en este sentido "el liderazgo al interior de la institución es vital para la conducción de la instituciones de educación superior hacia sus objetivos y para la realización de su misión". 38

En este contexto, es trascendental acoplar en las universidades un enlace permanente de entendimiento e interacción con el entorno y con los distintos sectores de la sociedad, ya que es imprescindible razonar sobre todo en los medios universitarios tradicionales, que la calidad en concreto no es una característica inherente a las instituciones de educación superior, sino que es un atributo otorgado por quienes sufren o se benefician del desempeño social y productivo de las universidades, de hecho, uno de los objetivos fundamentales de las políticas educativas de la actualidad, es la adaptación de las instituciones de educación superior a una perspectiva de evaluación de la calidad que se centra en la capacidad de resolución y de flexibilidad institucional para adecuarse y responder a las exigencias de las sociedades complejas de la información y del conocimiento,

37 Jorge Uribe, Lineamientos de política y guía práctica para la internacionalización de las universidades de la red: Por una internacionalización sostenible para el desarrollo regional y el avance de la calidad de la educación superior a través de las fronteras en el Eje Cafetero. (Universidad Tecnológica de Pereira, Serie de Documentos Académicos, RED ALMA MATER, 2009) http://www.almamater.edu.co/ sitio/Archivos/Documentos/Documentos/00000083.pdf (24 de septiembre de 2014)

38 Uriel Giraldo, Darío Abad, Edgar Díaz, Bases para una política de calidad de la educación superior en Colombia (Consejo Nacional de Acreditación -CNA, 2002) http://cms-static.colombiaaprende.edu.co/cache/binaries/articles-186502_doc_academico10.pdf?binary_rand $=8818$ (Septiembre 22 de 2014) 
incluso, para que las instituciones de educación superior puedan responder de manera adecuada y creativa a las exigencias que representa la continua variación e innovación de las demandas productivas y laborales de la actual sociedad del conocimiento, es necesario según el Consejo Nacional de Acreditación de Colombia:

Imperante adaptación de las universidades a un entorno cambiante debido a las transformaciones de los mercados ocupacionales, al aumento significativo del conocimiento avanzado, a la introducción de la tecnología de la información y la comunicación, a los cambios en los intereses profesionales de los jóvenes, y a las necesidades de los países de aumentar su competitividad. ${ }^{39}$

En este orden de ideas, uno de los elementos de mayor importancia de este nuevo contexto configurado por el impulso de los procesos de aseguramiento de la calidad, es el de la internacionalización de la educación superior, fenómeno con el que deben entrar a interactuar las universidades a través de los convenios de oferta de servicios educativos mixtos o compartidos, que van desde las dobles titulaciones a la movilidad estudiantil y docente, a las investigaciones co-participativas, y a la validación de créditos mutuos, entre otras actividades, que, de hecho, en la internacionalización de la educación en el ámbito de los procesos de aseguramiento de la calidad, encuentran el fundamento de su legitimidad y el nivel básico de su funcionamiento, en el desarrollo y en la organización de un campo intelectual y laboral.

En relación a la evaluación y aseguramiento de la calidad de la educación superior, que manifiesta uno de los factores de la consolidación del campo, en la actividad continua y productiva de los pares académicos internacionales, que no solo actúan en relación a la evaluación externa de la calidad, sino que realizan importantes aportes en factores cotidianos del mejoramiento de la calidad como lo es en la investigación y en la docencia sobre todo en los programas de postgrados de alto nivel de reconocimiento académico, de ahí qué, cobijados bajos éstas razones, los sectores más entusiastas de la internacionalización de la educación superior, contemplen que la experiencia desarrollada es suficiente como para pensar en que se poseen, los "elementos comunes entre países, para asentar los pilares que configuran un modelo mundial de aseguramiento de la calidad" ${ }^{40}$.

\section{CONCLUSIÓN}

En la actualidad se aprecia un contexto de reacomodo de los mercados nacionales e internacionales de educación superior, en relación a la introducción de nuevos sectores de provisión de servicios educativos aliados al capital financiero

39 Gloria Alzate. "Efectos de la acreditación en el mejoramiento de la calidad de los programas de psicología de Colombia", Universitas psychologica, vol. 7 no. 2 (2007): 114. En http://www.ascofapsi.org.co/observatorio/documentos/cap09_psychologica_V7N2.pdf

40 Gloria Alzate. "Efectos de la acreditación en el mejoramiento de la calidad de los programas de psicología de Colombia", Universitas psychologica, vol. 7 no. 2 (2007): 114. En http://www.ascofapsi.org.co/observatorio/documentos/cap09_psychologica_V7N2.pdf 
internacional, en una tendencia que configura, por un lado, contradicciones, en cuanto a la implantación de una competencia sin fundamentos territoriales, ni productivos, que conlleva una oferta de programas sin concordancia al mundo laboral real y nacional, pero, que, por otro lado, también configura complementaciones en relación a los convenios de innovación, de oferta de programas de doble titulación y de cooperación en investigación, que, al fin y al cabo, son la manifestación de una tendencia que se expresa en la tensión tradicional entre los intereses conservadores de las instituciones nacionales para conservar las exenciones públicas y las prerrogativas en el mercado del sector, frente a las expectativas liberales internacionales y transnacionales de apertura del sector de la educación superior, que es una situación que está apoyada por las orientaciones de liberalización de los mercados de educación superior, impulsados por organismos internacionales como el Banco Mundial, que claramente ubica las instituciones universitarias, como uno de los objetivo de sus intereses y de sus políticas de crecimiento económico, al promocionar la educación superior para el siglo XXI, como:

Un factor fundamental para los países en desarrollo, que buscan la inserción a la economía del conocimiento, donde se aconseja a los gobiernos garantizar el financiamiento a las instituciones públicas y contribuir a crear un clima conducente al financiamiento sustentable de las instituciones privadas, pensando en los sistemas de educación a largo plazo. ${ }^{41}$

Ahora, como rasgo específico del mercado de educación superior en América Latina, se puede observar, que si bien, existen nuevas exigencias laborales y sociales que la sociedad del conocimiento le impone a los sistemas de educación superior, que buscan ser resueltas a través de las políticas de aseguramiento de la calidad, no existen políticas, acuerdos u orientaciones consensuadas o compartidas respecto al carácter o el sentido de la implementación de los sistemas de aseguramiento de la calidad, aun cuando sea para enfrentar por parte de los sistemas de educación superior, desafíos institucionales que presentan similares características, presentándose por el contrario, que, aun cuando, "la preocupación por la calidad es un denominador común en los países latinoamericanos, es insuficiente la consolidación de los sistemas de aseguramiento de la calidad, debido a la heterogeneidad en aspectos legislativos, ligados al devenir político de cada país". ${ }^{42}$

En este sentido, se pueden identificar dos niveles de aplicación de las políticas de aseguramiento de la calidad de las que surgen diferenciaciones y heterogeneidad en la educación superior en el contexto latinoamericano, por un lado, la heterogeneidad en el nivel de las normativas que sustentan el aseguramiento de

41 Jorge Rodríguez. "Elementos para la discusión de la internacionalización de la educación superior colombiana", Revista de la Facultad de Ciencias Económicas de la Universidad Militar Nueva Granada, vol. 17 no. 1 (2009): 110. En http://www.umng.edu.co/www/ resources/Internacionalizacion.pdf

42 Gloria Alzate. "Efectos de la acreditación en el mejoramiento de la calidad de los programas de psicología de Colombia", Universitas psychologica, vol. 7 no. 2 (2007): 114. En http://www.ascofapsi.org.co/observatorio/documentos/cap09_psychologica_V7N2.pdf 
la calidad de la educación superior en cada país, y, por otro lado, en el nivel significativo y cotidiano de aplicación de la políticas de aseguramiento de la calidad en los procesos institucionales de cada universidad o de cada proveedor de servicios educativos, que, de hecho, en el primer nivel, en el de la heterogeneidad normativa, se refleja que, "los sistemas de educación superior latinoamericanos funcionan de manera muy distinta entre sí, y el tipo de normatividad que los rige, así como los organismos encargados de aplicarlas, son muy diferentes". ${ }^{43}$

Por el otro lado, en el nivel significativo y cotidiano de aplicación de las políticas de aseguramiento de la calidad, que es el de las instituciones universitarias, surge como un factor fundamental de diferenciación las limitaciones a la capacidad de flexibilidad institucional y organizativa que imponen las tradicionales y burocratizadas políticas educativas, que formulan su sustento normativo e histórico en las ideas de, "cogobierno y autonomía, que derivan en una modalidad de funcionamiento que restringe la formulación de políticas sistémicas para el conjunto de las instituciones de educación superior al supeditarlas a consensos difíciles dada la diversidad de misiones de la institución" ${ }^{44}$

En este orden de ideas, se puede determinar entonces, que uno de los rasgos que identifican los sistemas de educación superior en el contexto latinoamericano en el ámbito de las políticas de aseguramiento de la calidad, es la diversidad institucional que se configura en la actualidad, de ahí que, sirva de ilustración de este escenario, el caso colombiano, en el que la configuración del mercado de educación superior refleja la diversidad institucional, así como su ineficiencia en la dimensión de la organización del sistema de instituciones universitarias.

En la actualidad las políticas públicas educativas consolidan las directrices de la internacionalización y del aseguramiento de la calidad en el contexto de la integración del mercado educativo a nivel global, con tal fuerza política, empuje económico y sustento conceptual, que para un país como Colombia, los desafíos se concentran en los procesos de desarrollo institucional de las instituciones de educación superior, pues, éstas deben adquirir niveles de flexibilidad administrativa, docente e investigativa que se fundamenten más en la sistematización de la información y en la utilización inteligente de ésta información, pues, para la competencia mundial en el campo educativo, el sistema de educación superior colombiano aún no configura un conglomerado interinstitucional que movilice y empeñe la agenda pública y política con los recursos suficientes hacia el sector de la educación superior, entendido como un campo estratégico e imprescindible para enriquecer y mejorar los índices de desarrollo social y de competitividad económica del país y de sus regiones frente al escenario internacional.

43 Ernesto Villanueva. Aseguramiento de la calidad y convergencia de la educación superior en América Latina (Seminario Movilidad, Aseguramiento de la Calidad y Reconocimiento Mutuo de Títulos de Educación Superior en América Latina. Cátedra UNESCO Cinvestav, 2006). http://www.cna.gov.co/1741/articles-186502_Aseguramiento.pdf (Octubre 2 de 2014)

44 Claudio Rama. "La tercera reforma de la educación superior en América Latina", Revista Educación y Pedagogía, vol. 18 no. 46 (2003): 12. En http://aprendeenlinea.udea.edu.co/revistas/index.php/revistaeyp/article/viewFile/6875/6292 


\section{REFERENCIAS}

Alzate, Gloria. “Efectos de la acreditación en el mejoramiento de la calidad de los programas de psicología de Colombia", Universitas psychologica, vol. 7 no. 2 (2007): 114.

Aparicio, Pablo. "Educación y jóvenes en contextos de desigualdad socioeconómica". Revista Tendencias y perspectivas en América Latina. Archivos Analíticos de Políticas Educativas, vol. 17, no. 12 (2009): 1-35.

Arnaiz, José. “La Interrelación entre el Nivel Educativo y la Posición Competitiva en América Latina y la Unión Europea", Anales de Estudios Económicos y Empresariales, no. 20 (2010): $87-124$.

Correia, Manolita. "La internalización de la industria de la educación: El caso Australiano". Revista Calidad de la Educación, no. 32 (2010): 170-204.

Espinosa, Alberto, Empresarios y política pública educativa (Revista Dinero.com. 2009) http:// www.dinero.com/edicion-impresa/columnistas/empresarios-politica-publica-educativa_61202.aspx (23 octubre de 2014)

Espinosa, Oscar. "Reflexiones sobre los conceptos de política, políticas públicas y política educacional", Revista Archivos Analíticos de Políticas Educativas, vol. 17, no. 8 (2009): 1-13.

Fernández, Eduardo. “El sistema-mundo del capitalismo académico: Procesos de consolidación de la universidad emprendedora". Revista Archivos Analíticos de Políticas Educativas, no. 21 (2009): 1-43.

Giraldo, Uriel; Abad, Darío y Díaz, Edgar, Bases para una política de calidad de la educación superior en Colombia (Bogotá: CNA, 2002) http://www.cna.gov.co/1741/articles-186502_doc_academico10.pdf (6 de septiembre de 2013)

Giraldo, Uriel, Abad, Darío y Díaz, Edgar, Bases para una política de calidad de la educación superior en Colombia (Consejo Nacional de Acreditación -CNA, 2002) http://cms-static. colombiaaprende.edu.co/cache/binaries/articles-186502_doc_academico10.pdf?binary_rand $=8818$ (Septiembre 22 de 2014)

Gómez, Víctor, Celis, Jorge. “El Sistema de Aseguramiento de la Calidad de la Educación Superior: Consideraciones Sobre la Acreditación en Colombia". Revista Colombiana de Sociología. vol. 32 no. 2 (2009): 87-110.

González, Elvia y Duque, Sandra. "Sobre el marco normativo para formular una propuesta de políticas públicas para la formación por ciclos y la evaluación por competencias en la educación superior colombiana", Revista Opinión Jurídica, no. 14 (2008): 83-98.

Martín, José. "Los vínculos entre capital humano y la competitividad en América Latina". Revista Estudios Económicos de Desarrollo Internacional, no. 002 (2007): 82-102.

Mora, Oliver. "Las políticas educativas en América Latina: Un análisis de la educación superior desde la visión de la banca multilateral". Apuntes del CENES, vol. 25, no. 40 (2005): 249262.

Moreles, Jaime. “Uso de la investigación en la toma de decisiones políticas. Una aproximación a su estudio", Revista Archivos Analíticos de Políticas Educativas, vol. 17 no. 13 (2009): 1-25.

Rama, Claudio. "La tercera reforma de la educación superior en América Latina", Revista Educación y Pedagogía, vol. 18 no. 46 (2003): 11-24.

Rivera, Felipe, Astudillo, Pablo y Fernández, Enrique. “Información y toma de decisiones: Oficinas de análisis institucional (OAI)". Revista Calidad de la Educación, no. 30 (2009): 162178.

Rodríguez, Jorge. “Elementos para la discusión de la internacionalización de la educación superior colombiana", Revista de la Facultad de Ciencias Económicas de la Universidad Militar Nueva Granada, vol. 17 no. 1 (2009): 119-122. 
Soto Arango, Diana Elvira . "Legislación e imaginarios sociales en el escalafón y los salarios de los educadores de primaria en Colombia 1952 - 1994" Revista Historia de la Educación Latinoamericana no. 21 (2013): 229-262

Scharager, Judith y Aravena, María. “Impacto de las políticas de aseguramiento de la calidad en programas de educación superior: Un estudio exploratorio". Revista Calidad de la Educación, no. 32 (2010): 16 - 42.

Uribe, Jorge. Lineamientos de política y guía práctica para la internacionalización de las universidades de la red: Por una internacionalización sostenible para el desarrollo regional y el avance de la calidad de la educación superior a través de las fronteras en el Eje Cafetero. (Universidad Tecnológica de Pereira, Serie de Documentos Académicos, RED ALMA MATER, 2009) http://www.almamater.edu.co/sitio/Archivos/Documentos/Documentos/00000083.pdf (24 de septiembre de 2014)

Villanueva, Ernesto. "Perspectivas de la educación superior en América Latina: Construyendo futuros". Revista Perfiles Educativos. no. 129 (2010): 86-101.

Villanueva, Ernesto, Aseguramiento de la calidad y convergencia de la educación superior en América Latina (Seminario Movilidad, Aseguramiento de la Calidad y Reconocimiento Mutuo de Títulos de Educación Superior en América Latina. Cátedra UNESCO - Cinvestav, 2006). http://www.cna.gov.co/1741/articles-186502_Aseguramiento.pdf (Octubre 2 de 2014)

Zambrano, Carmen y Benítez, Gonzalo. "Laboratorio de análisis institucional del sistema universitario mexicano (LAISUM)". Revista Perfiles Educativos, vol. 32, no. 129 (2010): 184186. En: http://www.iisue.unam.mx/seccion/perfiles/

Zapata, Gonzalo, Tejada, Ivo. "Impactos del aseguramiento de la calidad y acreditación de la educación superior. Consideraciones y proposiciones", Revista Calidad de la Educación, no. 31 (2009): 192-209.

Cómo citar:

Malagón Plata, Luis Alberto; Rodríguez Rodríguez, Luz Helena;

Machado Vega, Diego Fernando. "Políticas Públicas Educativas y aseguramiento de la calidad en la Educación Superior" Revista Historia de la Educación Latinoamericana. Vol. 21 No. 32 (2019): 273-290 https://doi.org/10.19053/01227238.4999

(c) (1) (-) Esta obra está bajo una licencia Creative Commons. Reconocimiento-No Comercial-Sin Obra Derivada 2.5 Colombia. 